\title{
Editorial
}

\section{Geoffrey Samuel}

One of the highlights of the 2009 IASTAM Conference in Bhutan was the presentation of the Basham Medal. The award was instituted in the 1980s, and commemorates the name of Professor Arthur Llewellen Basham, cofounder of the International Association for the Study of Traditional Asian Medicine, of which this is the house journal. The Basham award is given "to recognize special contributions by IASTAM members to promoting the goals of IASTAM"; this was the sixth time that an award has been made. The recipient in Bhutan was Professor Vincanne Adams, of the Department of Anthropology, History and Social medicine of the University of California, San Francisco. In accordance with custom, she presented an address at the award ceremony, and this is the first item in the present issue.

Adams' essay is characteristic of her work, which is informed by recent postcolonial debates in science and technology studies, and has always been ready to challenge biomedical understandings of health and the human body in favour of the more expanded and inclusive view that she sees in Tibetan medicine and other Asian medical systems. She is also a scholar who, while engaging with her topic at the highest professional levels, recognises the importance of the heart as well as that of the intellect, a point made cogently in her opening story of her meeting with His Holiness the Dalai Lama.

Adams' topic, 'Efficacy,' is of course at the centre of many of the debates and discussions in our field. Adams sees exploring efficacy as 'an empirical problem just as much for the shaman as for the pharmaceutical scientist, and as an endeavour that can contribute social science insights to an understanding of how best to heal'. She confronts the relationship between biomedical and nonWestern modes of healing head-on, insisting that as long as we insist on confining efficacy in non-Western medicine to those elements that can be explained in biomedical terms, we may miss what is really happening in situations of healing, or of failure to heal. Efficacy, she argues, is a floating signifier, and there is no firm ground on which it can rest. Her essay ends with a question 
that few people working seriously in this field can evade, and which ultimately has no easy answers: what is healing?

The ICTAM conference in Bhutan was a remarkable academic event in many respects, and it has generated a series of special issues, in which the kind of concerns Adams outlines often feature in one way or another. Our last issue, 5.2, was the first of these special issues, and two more are planned for the near future. The remainder of the present issue, however, is taken up with a series of articles on varied themes, only one of which (that by Paul Kadetz and Johann Perdomo Delgado) originated in a paper at the ICTAM conference. Issues raised at the conference, including those stated so trenchantly in Adams' paper, can nevertheless be seen in many of these articles.

Fred Smith's article on sattvāvajaya is one of three primarily textual and historical articles. Like several of his recent writings, it links to the concerns of his magisterial volume The Self Possessed (Columbia University Press, 2006) which explored vocabularies of possession throughout South Asia, both in textual and ethnographic sources. Sattvävajaya is a medical term used in one of the Indian medical classics, the Caraka Samhitā, where it describes one of three primary categories of treatment. The term has never been satisfactorily explained. Smith suggests that it refers to a form of therapy involving spirit possession, such as has been witnessed from a range of textual and ethnographic evidence. Such practices, he suggests, have a 'deep history' within Indian culture. He speculates as to why, if this were so, the later medical tradition failed to recognise them, suggesting that they were 'marginalised to the point of elimination' because they ran counter to the 'scientific, or at least empirical, credibility' that Indian medical writers sought. The parallels here to Adams' essay are indeed quite evident.

Pierce Salguero's article is also concerned with Indian medical terminology, but explores its translation into Chinese sources. Translations on Buddhist texts into Chinese necessarily include reference at times to medical terminology. To the extent that the translations of medical terminology have been considered in the past, they have generally been regarded as confused and inaccurate. Salguero suggests that it is worth looking more closely at these translations, particularly in relation to the terms for the three doșa or pathogenic principles. He argues that the ways in which these terms were translated reflect processes of cultural negotiation, in which the Chinese translators were attempting to arrive at versions that made sense within the logic of Chinese medical thought. Their aim was to make the foreign medical doctrines comprehensible to their Chinese readers. Salguero's article is a contribution to a small but significant and growing body of studies that looks at the processes of transformation between medical concepts as they pass between cultures. 
Francis Garrett returns us to the Tibetan territory of Adams' Basham Prize essay, and to the complex interface between medical and religious practices in Tibet, as well as to another 'fuzzy boundary,' that between medicine and food. Her topic is the use of food in various Tibetan ritual practices. Food here includes the well-known torma or ritual cakes used in many Tibetan rituals for healing and other purposes, which are in effect food offered to the deities. A critical component is the ritual empowerment and transmutation of the ritual cakes into nectar (bdud rtsi, corresponding to Skt. amrta). 'Nectar medicine' is not only prepared for the deities, however; similar techniques are used to empower substances as food and medicine for personal consumption. A wide range of pills and other substances believed to be both powerful and efficacious are prepared in this way, and a large literature has developed on this topic in Tibet. Garrett asks how best to frame and understand what is going on in these procedures, suggesting that studying the 'culinary aesthetics' underlying the making of these ritual foods and medicines might help us understand why they are thought to be powerful and effective.

Leigh Chipman and Efraim Lev's article examines mediaeval Arabic medicine on the basis of medical prescriptions found in the Cairo Genizah. These prescriptions present a valuable alternative perspective to the pharmacopoeias on which has been based most previous research on Arabic pharmacy and pharmacology, giving a valuable witness to medicine as actually practised in mediaeval Cairo. The article examines a number of prescriptions in detail, with the aim of understanding who wrote them, who prepared the recipes, what can be learnt from them about medical practice at the time. The substantial presence of materia medica from India and China is a point of particular interest. While the recipes and ingredients are similar to those in the contemporary pharmacopoeias, some of which also survived in the Genizah collection, they are never identical, suggesting 'the flexibility of the medical treatment offered to patients in medieval Cairo, which may have been tailored to individual patients on the basis of known recipes, rather than the physician choosing from among pre-existing drugs (as a modern practitioner of bio-medicine must do)'.

Paul Kadetz and Johann Perdomo Delgado's article moves us to a more contemporary context. This article examines how Chinese medicine became an integral part of the Cuban health system. Kadetz and Perdomo give due attention to similarities between Chinese herbal medicine and acupuncture and already-present local healing practices, but the primary frame of reference here is political economy. An effective universal health care system was one of the key goals of the revolutionary Cuban state, which made significant progress in this direction despite the US blockade. TCM, including both 
acupuncture and the extensive use of locally-available herbal medicines adapted to Chinese principles, became institutionalised in Cuba at time when the economic collapse of the former Soviet Union removed Cuba's main remaining source for pharmaceutical raw materials. Thus non-biomedical health care was embraced out of necessity, as a way to continue to deliver effective health care despite the difficult circumstances. Kadetz and Perdomo note how this has affected the character of the Cuban version of Chinese medicine. Acupuncture is learnt on cadavers, not on live bodies, so the sensitivity to $q i$ that is a hallmark of traditional practice is lost, and the logic behind acupuncture is strongly biomedicalised. Yet, by 2002 , ' $86 \%$ of Cuban physicians were practising some form of MTN' (traditional and natural medicine), and a high proportion of pharmaceuticals had been replaced by plant medicines. Cuba offers one of the most effective examples of the incorporation of an Asian medical tradition into a modern Western state.

Phurpa Wangchuk and Annette Olsen's article is also about the practicalities of mainstreaming traditional medicine in a small modern state, in this case Bhutan. Sowa rigpa or Tibetan medicine was present in pre-modern Bhutan, but on a small scale, effectively as an elite medical tradition carried out by a few Tibetan-trained doctors under aristocratic patronage. The modern Kingdom of Bhutan has cultivated and supported sowa rigpa alongside biomedicine. A medical college and hospital is situated close to the capital, Thimphu, and the aim is that sowa rigpa will be made available throughout Bhutan alongside biomedicine. Yet scaling up the provision of sowa rigpa has implications for the supply of traditional medicines, and medicinal plants are vulnerable to the impact of modernity and the capitalist economy in other ways as well. Wangchuk and Olsen discuss the risks to the sustainability of medicinal plants in Bhutan and also the measures which have been taken to control plants and where possible to cultivate them. This article adds further significant data to the essays on the conservation, cultivation, and commodification of medicinal plants in the greater Himalayan-Tibetan Plateau that were presented in the last issue, the special issue on this theme edited by Sienna Craig and Denise Glover. Bhutan is the state in the region with much the strongest commitment to the preservation and support of sowa rigpa, so its experience is of particular interest.

Anthony Butler and Tina Huang's review of studies of acupoint stimulation brings us in some ways back to the ground of Adams' article, the contested relationship between Asian and other non-biomedical modalities of healing and the dominant biomedical tradition. Butler and Huang examine the contribution of magnetic resonance imaging (MRI) to the somewhat vexed issues of the effects of acupuncture on areas of the body distant from the points 
needled, and, particularly, the difference between needling at 'genuine' acupoints and at 'sham' or non-standard locations. Since MRI provides a way to record the organism's response independent of subjective reporting, it offers an opportunity to bypass some of the practical and methodological problems involved in earlier studies. However, MRI is far from a straightforward technology, as Butler and Huang's account make clear, and the results so far are less than fully convincing. One result is that it is clear that needling at the 'traditional' acupoint at best 'optimises an effect that can be elicited by needling over quite an area'. This is perhaps only to be expected, since, as Vivienne Lo notes, past practitioners of acupuncture and moxibustion for the most part did not use precise locations for the point system, which is notoriously inexact. In the light of the considerable effect attributed to using non-standard locations, research geared towards establishing a difference between 'true' acupoint stimulation and placebo treatment would seem somewhat misguided.

The impact of history, politics and political economy has figured directly or indirectly in several of these articles. Who has access to medicine, why particular schools and varieties of medicine are promoted and others not, and how we as scholars confront the location of medicine and healing in a world of unequal access to limited resources are questions which are necessarily woven through much of the work in our field, particularly in relation to contemporary times. We are particularly pleased to be present an interview by Rohan Deb Roy with David Arnold, a leading historian of colonial and modern medicine in South Asia, as the last article in the issue, since this interview foregrounds and discusses in detail many of these questions. Our field needs much close and detailed work, but it also needs to retain an awareness of the wider context within which specific studies are located. Arnold's writings are a model of how this can be done, and we trust that our readers will find this interview enlightening and thought-provoking.

The issue is rounded off with two substantial book reviews, one of a significant recent ethnography of Chinese medicine in China and the USA, the other of an important recent collection on modern and global Ayurveda. We hope that all our readers will find plenty to interest them in this wide-ranging and stimulating collection. 